\title{
ASSURANCE SANTE: UN MOYEN DE FINANCER LA SANTE POUR TOUS \\ L'EXPERIENCE DU CENTRE DE DEVELOPPE- MENT INTEGRAL DE BWAMANDA, ZAIRE
}

\section{SEMA HURUGO}

\section{CONTEXTE}

Le Centre de Développement Intégral de Bwamanda (C.D.I. -Bwamanda) est un programme multisectoriel qui a débuté ses activités de développement en 1969 au Nord-Ouest du Zaïre.

Globalement le C.D.I.-Bwamanda mène deux activités principales:

* des activités agro-économiques: vulgarisation agricole auprès des paysans. Le but de ces activités est de donner un revenu aux paysans afin de leur permettre de subvenir à certains besoins (scolarisation des enfants, soins médicaux, etc.)

* des activités médico-sociales: gestion de 3 districts sanitaires (avec 5 hôpitaux et 80 centres de santé), service de développement rural qui s'occupe de l'animation, mobilisation de la population encadrée par le C.D.I. -Bwamanda.

L'accessibilité financière de la population aux soins de santé est une des préoccupations majeures du C.D.I.-Bwamanda. 


\section{ORIGINE ET CONCEPTION DE L'ASSURANCE-SANTE}

Le financement communautaire des soins de santé au niveau des centres de santé (1er échelon du système sanitaire au Zaïre) est une option acceptée au Zaïre en général et dans le district de Bwamanda (Nord-Ouest du Zaïre) en particulier. Les prix des soins de santé sont abordables à ce niveau.

Cependant au niveau des hôpitaux le coût élevé des prestations (techniques) ne permet pas à la population de payer. De plus, l'incapacité des pouvoirs publics à assurer le financement des charges récurrentes des hôpitaux pose des problèmes pour leur fonctionnement.

Conscients de ce problème, les responsables du district sanitaire de Bwamanda ont mis sur pied, depuis 1986, un système de prépaiement des soins hospitaliers (à l'hôpital de référence du district sanitaire).

Trois raisons ont motivé la mise en place de ce système:

- on a constaté avant 1986 que les habitants du district sanitaire éprouvaient des difficultés à payer les soins hospitaliers

- les paysans qui vivent de leurs récoltes n'ont pas de revenus stables pendant toute l'année; il fallait concevoir un système leur permettant un accès permanent à l'hôpital

- les sources de financement extérieur ont diminué.

Au départ, des discussions ont eu lieu entre les comités de santé, les infirmiers des centres de santé, les agents du service de développement rural ainsi que l'équipe-cadre du district sanitaire.

De ces discussions, un modèle d'Assurance-Santé (Mutuelle) adapté à la situation locale a été mis sur pied avec la participation active de la population. C'est depuis 1986 que l'Assurance-Santé (appelée "mutuelle") existe dans le district de Bwamanda (un des 3 districts sanitaires gérés par le C.D.I.-Bwamanda). 


\section{LES OBJECTIFS DE LA MUTUELLE}

Ce système d'Assurance-Santé a 3 objectifs:

- améliorer l'accessibilité financière de la population du district sanitaire aux soins hospitaliers

- améliorer la situation financière de l'hôpital

- apprendre à la population couverte par les activités du district sanitaire à planifier l'utilisation de ses revenus, à gérer le futur.

La mutuelle permet ainsi à la population participante de financer les soins hospitaliers de ceux qui ont la malchance de tomber malades. Ceci favorise un partage des risques de maladie entre les malades et les bien-portants et assure une promotion de la solidarité entre les gens.

\section{ORGANISATION ET GESTION}

L'enregistrement des membres de la mutuelle se fait au mois de mars chaque année. Cette période coïncide avec la campagne d'achat des produits agricoles. Le paysan dispose à ce moment d'un revenu.

L'enregistrement est précédé d'une sensibilisation de la population au cours de laquelle on explique que la maladie est un risque inhérent à la vie et qui pose un certain nombre de problèmes inquiétants, parce que son apparrition, sa gravité, sa durée, son coût global et ses conséquences socio-économiques, professionnelles ou morales sont imprévisibles. D'où la nécessité d'adhérer à la mutuelle.

L'enregistrement se fait au niveau de chaque centre de santé où sont tenues des fiches de la population. Ces fiches indiquent la composition de la famille ainsi que les épisodes de la maladie significatifs de chaque famille. La prime à payer est annuelle. Elle est fixée par l'équipe-cadre du district en tenant compte $d u$ pouvoir d'achat de la population. Le prix est fixé empiriquement et équivaut à environ $2 \mathrm{~kg}$ de soja ou $4 \mathrm{~kg}$ de maïs par an et par individu. 
En cas de maladie, le membre de la mutuelle se présente à l'hôpital muni d'un bon de transfert de son centre de santé. Il paie alors $20 \%$ du tarif des non-membres de la mutuelle et bénéficie d'une réduction de $80 \%$ des tarifs en vigueur.

Chaque année, $60 \%$ de la population du district souscrivent à la mutuelle. L'argent collecté est investi dans les médicaments afin d'éviter toute diminution de la valeur des fonds recouvrés sous l'effet de l'inflation et de la dévaluation.

La comptabilité de la mutuelle est tenue séparément et l'hôpital facture la mutuelle pour les dépenses encourues par les assurés.

\section{CONDITIONS D'AFFILIATION A LA MUTUELLE}

Tous les habitants du district de Bwamanda peuvent souscrire à la mutuelle. L'enrôlement n'est pas obligatoire mais tout individu qui s'inscrit doit faire inscrire également tous les membres de sa famille. Donc l'unité d'enrôlement est la famille. Cependant, le coût d'enrôlement est fixé par tête et non par famille.

Autres conditions d'affiliation:

- $\quad$ etre recensé dans le district, c.à.d. être inscrit dans son centre de santé

- la cotisation est annuelle mais la période d'enregistrement ne couvre qu'un mois (le mois de mars, période où la production agricole est achetée aux paysans par le C.D.I.-Bwamanda).

- en cas d'hospitalisation, la mutuelle n'intervient que si le malade a été référé par son centre de santé. Ceci évite une surutilisation (irrationnelle) du système de mutuelle.

La mutuelle couvre tous les soins au niveau de l'hôpital et quelques soins primaires au niveau du centre de santé (maladies chroniques (e.a. tuberculose, maladies cardiaques, lèpre) et accouchements pour les membres affiliés.

\section{FACTEURS DE SUCCES}

Différents facteurs ont conditionné le succès de la mutuelle à Bwamanda:

(1) La participation de la population et des agents du Service Médical et du Développement Rural dans toutes les phases de la mutuelle: conception, 
choix du modèle et organisation. La participation de la population est suscitée par toutes les activités de développement menées par le C.D.I.-Bwamanda.

(2) L'existence d'une relation de confiance entre la population et le Service Médical. Cette confiance est due à la qualité des soins offerts à la population et à toutes les autres actions de développement du C.D.I.-Bwamanda (activités agricoles, forage de puits d'eau potable, animation rurale, ...).

(3) La possibilité d'investir l'argent collecté lors de l'enrôlement dans l'achat des médicaments. L'existence d'une pharmacie régionale bien gérée permet de maintenir la valeur des fonds collectés.

\section{CONCLUSIONS ET PERSPECTIVES D'AVENIR}

L'Assurance-Santé est une bonne illustration de ce qui peut être réalisé dans un programme de développement intégré, basé sur une approche intersectorielle où plusieurs actions sont entreprises dans divers secteurs. Elle nécessite un dialogue permanent entre la population et les agents de santé à tous les niveaux.

La mutuelle initiée à Bwamanda a favorisé:

(1) L'amélioration de l'accessibilité financière de la population aux soins. Elle couvre $80 \%$ du prix forfaitaire d'une hospitalisation ou du traitement ambulatoire d'une maladie chronique au niveau des Centres de Santé.

(2) L'amélioration financière de l'Hôpital Général de Référence, en assurant un recouvrement des coûts de $70 \%$. Ceci n'est pas facile à réaliser dans un environnement caractérisé par une crise socio-économique comme celle $d u$ Zaïre actuel.

(3) La promotion de la solidarité entre les habitants du district sanitaire. Les bien-portants payent pour ceux qui ont la malchance de tomber malades.

La viabilité de la mutuelle dépend de l'acceptation de ce système par la population et du contexte économique. A Bwamanda, la population a compris le bien-fondé et les avantages de la mutuelle. Ce qui constitue déjà un atout majeur pour la survie de la mutuelle dans le district de Bwamanda. L'exemple de Bwamanda peut servir aux autres districts du Zaïre qui sont confrontés aux problèmes de recouvrement du coût des soins de santé. La généralisation de la mutuelle dans les différents districts du Zaïre permettra d'améliorer la viabilité du système de santé basé sur les Soins de Santé Primaires et l'état de santé de la population zaïroise. 


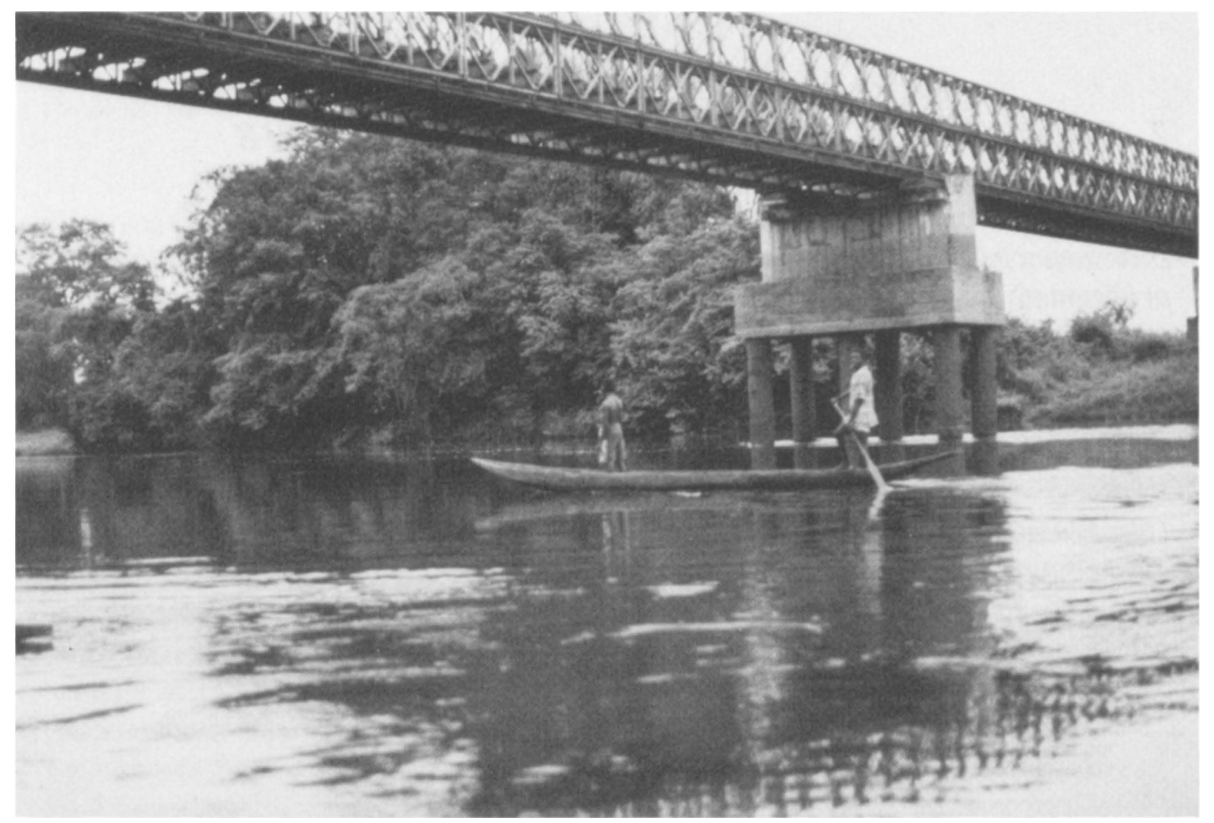

Brug over de Lua, gebouwd door C.D.I.-Bwamanda

Pont de la Lua, construit par le C.D.I.-Bwamanda 